\title{
Babesiosis in Pregnancy: An Imitator of HELLP Syndrome
}

\author{
Raminder Kaur Khangura, MD ${ }^{1}$ Nayo Williams, MD ${ }^{1} \quad$ Shontreal Cooper, MD, MPH \\ Anne- Marie Prabulos, MD ${ }^{1}$ \\ ${ }^{1}$ Division of Maternal Fetal Medicine, Department of Obstetrics and \\ Gynecology, University of Connecticut School of Medicine, \\ Farmington, Connecticut \\ Address for correspondence Raminder Kaur Khangura, MD, Division \\ of Maternal Fetal Medicine, Department of Obstetrics and \\ Gynecology, University of Connecticut, Farmington, CT 06032 \\ (e-mail: khangura@uchc.edu).
}

Am J Perinatol Rep 2019;9:e147-e152.

\section{Abstract Keywords \\ - HELLP imitators \\ - tick-borne illness \\ - pregnancy \\ - infectious disease \\ - babesia microti \\ - babesiosis}

HELLP syndrome (hemolysis, elevated liver enzymes, and low platelets) is a serious pregnancy complication that can cause significant maternal and neonatal morbidity and mortality. There are several conditions that may occur in pregnancy that may imitate the laboratory findings and clinical presentation of HELLP syndrome. Babesiosis is a parasitic imitator of HELLP syndrome that can be spread by the tick, transfusions, or congenitally. Recognition and treatment of this condition is important to optimize maternal and fetal outcomes.
Babesiosis is an infectious disease caused by the tick-borne protozoa, Babesia. ${ }^{1}$ Babesia microti, spread by the Ixodes scapularis tick, is the primary infectious agent causing babesiosis in the United States. ${ }^{2}$ It is endemic to the Northeast and upper Midwest, especially in parts of New England, New York, New Jersey, Wisconsin, and Minnesota. ${ }^{2}$ In 2014, 1,731 cases were reported to the Centers for Disease Control and Prevention (CDC) from 31 states, with sporadic cases reported outside of endemic areas in places, such as California, Alabama, and Washington. ${ }^{2}$

The expansion of the white-tailed deer population, wildlife habitat encroachment and greater awareness of the disease by physicians and the public have all contributed to the increasing numbers of Babesiosis. ${ }^{3}$ Clinical manifestations can vary from asymptomatic to life-threatening disease. In pregnancy, babesiosis can imitate HELLP (hemolysis, elevated liver enzymes, low platelets) syndrome., ${ }^{4,5} \mathrm{~A}$ high index of suspicion is needed to appropriately diagnose and treat HELLP imitators, such as Babesiosis, in pregnancy.

\section{Transmission}

\section{Tick-borne}

Although adult ticks can transmit B. microti, most cases are result from exposure to I. scapularis nymphs ( - Fig. 1) during late spring through summer. ${ }^{3}$ Tick-borne transmission of
Babesia primarily peaks during warm months and has been on the rise in the last decade, becoming almost as common as Lyme disease in some areas of southern New England. ${ }^{2,3}$ Symptoms usually occur 1 to 4 weeks after an infected tick bite, ${ }^{3}$ although many patients infected with babesiosis cannot recall a tick bite. ${ }^{2}$

\section{Blood Transfusion}

Babesiosis is currently the most common red blood cell (RBC) transfusion-transmitted pathogen reported to the U.S. Food and Drug Administration (FDA). ${ }^{6}$ Transfusion-transmitted babesiosis has been linked various blood products including RBCs, frozen deglycerolized RBCs, and whole blood-derived platelet concentrates (presumably from residual RBCs). ${ }^{6}$ This method of transmission may be one explanation for cases to occur in nonendemic areas because (1) patients with subclinical infection may donate blood in nonendemic areas and (2) infected blood may be exported to nonendemic areas in times of shortages. ${ }^{3,6,7}$ Patients with transfusion of contaminated blood products usually become symptomatic 1 to 9 weeks after transfusion with a median incubation period of 34 days. ${ }^{3,6}$

In a highly endemic area, some blood collection agencies have implemented investigational testing protocols for blood donor testing which helped in removing infected units from blood supply. ${ }^{6,8}$ In March 2018, the FDA approved the received

November 29, 2018

accepted after revision

December 11, 2018
DOI https://doi.org/

10.1055/s-0039-1687873. ISSN 2157-6998.
Copyright $\odot 2019$ by Thieme Medical Publishers, Inc., 333 Seventh Avenue, New York, NY 10001, USA. Tel: +1(212) 584-4662.
License terms

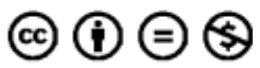




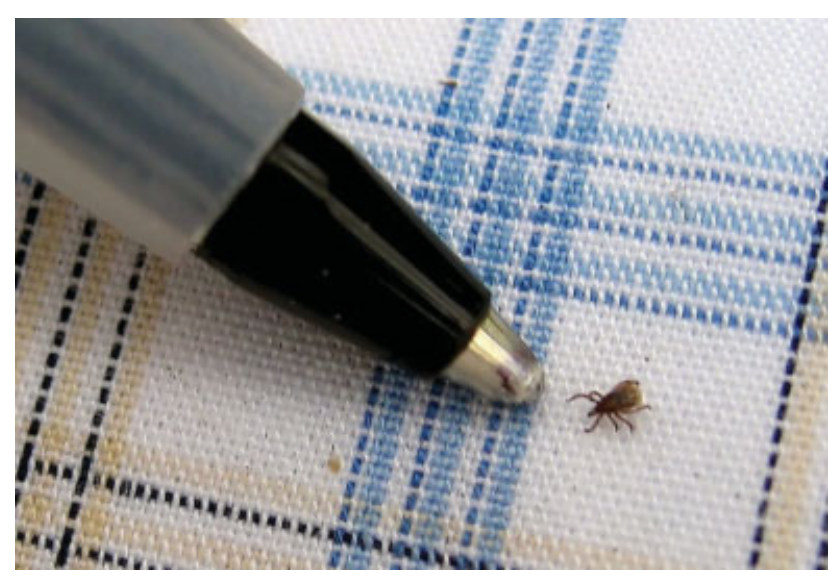

Fig. 1 Ixodes scapularis tick in lymph stage. Image provided by Dr. Graham Hickling, University of Tennessee, Institute of Agriculture.

Imugen $B$. microti arrayed flurosecent immunoassay (AFIA) for detection of antibodies to $B$. microti in human plasma samples and Imugen $B$. microti nucleic acid test (NAT) for detection in human whole blood samples. ${ }^{8}$ Approval of both tests was granted to Oxford Immunotec, Inc and are in house tests that can only be formed at the Norwood, Massachusetts facility. Currently, there is no guidance for testing of donor samples; however, the FDA is planning on issuing a draft guidance later this year with recommendations for reducing the risk of transfusion-transmitted babesiosis. ${ }^{8}$

\section{Transplacental}

Transplacental and congenital babesiosis has been documented in the literature. ${ }^{9}$ Cases of congenital babesiosis were detected 19 to 41 days after birth and were characterized by asymptomatic maternal infection, maternal fever, neonatal hemolytic anemia, and thrombocytopenia. Infants responded to antibiotic therapy and all infants required blood transfusion. ${ }^{10}$

\section{Pathogenesis}

Babesia organisms enter the erythrocyte and mature into merozoites which then invade nearby red cells, ensuring persistence of the infection. Pathogenesis is dependent on the host response to infection and parasite-induced alterations in the erythrocyte membrane. ${ }^{3,11}$ In mild cases of babesiosis, inflammatory cytokines, adhesion molecules, and vascular cell adhesion molecules are upregulated. ${ }^{3}$ In severe disease, excessive cytokine production may impair host mitochondrial function leading to tissue hypoxia, decreased erythrocyte deformability, and parasite killing causing severe infection and complications. ${ }^{12}$ Invasion and destruction of the erythrocytes can manifest as many other complications of babesiosis including fever, anemia, jaundice, hemoglobinemia, hemoglobinuria, and metabolic acidosis. Notably, this understanding of the varied host response in human subjects is limited and based on case studies, natural vertebrate hosts, and animal models. ${ }^{11}$ In mouse models, the protection conferred by adoptive transfer of splenic immune cells is age dependent and genetically determined. ${ }^{11}$

\section{Clinical Presentation}

B. microti infection can vary in clinical manifestation from asymptomatic to acute and fatal disease. ${ }^{7}$ As previously mentioned, presentations vary and depend on the host's immune status. ${ }^{5}$ In one cohort study, $20 \%$ of adults were asymptomatic. ${ }^{11}$ Patients with clinical manifestations can present with mild to moderate disease or severe disease. ${ }^{3}$ Asymptomatic and mild infections usually occur in patients who are immunocompetent and have parasitemia $<4 \%{ }^{1}$ Symptoms of mild/moderate disease are similar to common viral illnesses with gradual onset of malaise and fatigue. ${ }^{1,11}$ Fever usually develops with temperatures as high as $105.6^{\circ} \mathrm{F}$. Other common symptoms include chills and sweats and may be accompanied by a headache, myalgia, anorexia, nonproductive cough, arthralgias, and nausea. ${ }^{11}$

Patients affected with severe infection often have nausea, vomiting, diarrhea, and hemoglobinuria. ${ }^{7}$ Severe derangements in laboratory values can lead to a variety of complications and require hospitalization. Also, a parasitemia level $>4 \%$ is associated with severe disease. Risk factors associated with severe disease are parasitemia $>4 \%$, neonates, age of 50 years old and immunocompromised persons, such as those with cancer, human immunodeficiency virus infection, hemoglobinopathy, functional asplenia, chronic heart, lung, or liver disease. ${ }^{3}$ Pregnancy may predispose to increased severity of disease since it is an immunocompromised state; however, maternal risk factors for severe babesiosis are not well understood. ${ }^{4,13}$ However, it is important to note that there is not a strict relationship between the level of parasitemia above $4 \%$ and the severity of illness. $^{12}$

In both mild and severe disease, fever is the most common sign of babesiosis infection and maybe occasionally accompanied by splenomegaly or hepatomegaly. ${ }^{3,11}$ Although less common, mild pharyngeal erythema, jaundice, retinopathy, and retinal infarcts are also sometimes present. ${ }^{3} \mathrm{~A}$ rash is not usually present; however, if noted should raise the concern for coinfection with Lyme disease. ${ }^{3,11}$ Physicians should keep babesiosis on their list of differentials for febrile transfusion reaction, especially for the elderly, immunocompromised, and patients who have undergone a splenectomy. ${ }^{7}$

\section{Complications}

About $50 \%$ of patients hospitalized with babesiosis develop complications. ${ }^{11}$ The most common complications are acute respiratory distress syndrome and disseminated intravascular coagulation. ${ }^{11,14}$ Other complications, such as congestive heart failure, coma, liver failure, renal failure or splenic rupture, may also occur. ${ }^{11}$ Fatality rates are 6 to $9 \%$ amongst hospitalized patients and up to $21 \%$ in those patients with immunosuppression. ${ }^{11}$

When comparing nonpregnant patients with complications, severe anemia defined as a hemoglobin level $\leq 10 \mathrm{~g} / \mathrm{dL}$, were associated with complicated babesiosis. ${ }^{14}$ Parasitemia level $>10 \%$ was associated with complicated babesiosis but the association did not reach statistical significance. ${ }^{14}$ 


\section{Diagnosis}

A high index of suspicion is required to make a diagnosis of babesiosis. ${ }^{7}$ A diagnosis should be considered if a patient resides in or has traveled to a Babesia endemic area or has received a blood transfusion in the last 6 months and presents with symptoms that might be consistent with babesiosis. ${ }^{3}$ Diagnosis of babesiosis is by visualization of intraerythrocytic trophozoites or merozites on Wright-Giemsa stained blood smears under oil immersion. ${ }^{7}$ The level of parasitemia is generally between 1 and $10 \%$ but can be as high as $80 \%{ }^{3}$

Polymerase chain reaction (PCR) is a more sensitive blood test compared with a blood smear and provides molecular characterization of Babesia species. ${ }^{3}$ PCR can be considered of diagnostic benefit when the patient may be early in the phase of the infection and parasites may be difficult to visualize on blood smears. ${ }^{15}$ Serology is a useful tool for supporting the diagnosis; however, it may be absent in early disease and thus doesn't replace microscopy or PCR. ${ }^{15}$ A 4fold rise in Babesia IgG (immunoglobulin G) titer helps to differentiate between recent or past infection which a single positive antibody titer cannot do. ${ }^{3}$ During the acute phase of the illness, IgG titers can exceed 1:1,024 which usually decline to $1: 64$ or less within 8 to 12 months. $^{3}$

About $1 \%$ of patients infected with babesiosis have coinfection with another tick-borne disease, including anaplasmosis, ehrlichiosis, and Lyme disease. ${ }^{4}$ Thus, it is important to test for other tick-borne agents when presented with a presumed tick-borne disease. $^{7}$

\section{Laboratory Abnormalities}

Laboratory findings from the invasion and destruction of RBCs can lead to hemolytic anemia and can render laboratory abnormalities including low hemoglobin, low hematocrit, and elevated lactate dehydrogenase. Serum liver enzyme concentrations are often elevated, and thrombocytopenia is common. Severe illness may cause elevated serum levels of blood urea nitrogen and creatinine and are often accompanied by proteinuria. ${ }^{3}$

The laboratory findings of babesiosis are similar to HELLP syndrome and can often lead to a diagnostic dilemma. ${ }^{4}$ The clinical course of HELLP syndrome, like babesiosis, is often characterized by progressive and sometimes with a sudden deterioration. Thus, management of patients with preeclampsia or HELLP syndrome with worsening laboratory findings includes prompt delivery if beyond 34 weeks of gestation or earlier if there is disseminated intravascular coagulation, liver infarction, hemorrhage, renal failure, pulmonary edema, placental abruption, or nonreassuring fetal status. ${ }^{16}$

The differential diagnosis of babesiosis should be considered on the differential of HELLP syndrome imitators to avoid iatrogenic preterm delivery, delay in treatment, and complications of undiagnosed and untreated babesiosis. ${ }^{17}$ - Table 1 demonstrates the similar clinical manifestations and laboratory evaluation in HELLP syndrome and babesiosis.

A peripheral blood smear, which is typically done to evaluate for hemolysis in patients with HELLP syndrome, may aid in the diagnostic process by detecting intraerythrocytic parasites. ${ }^{5}$

\section{Babesiosis in Pregnancy: Case Series}

In a review of the literature and our institution's experience with babesiosis, we identified eight cases in pregnancy found in - Table 2. Two of these patients never had a fever. ${ }^{5,17}$ HELLP syndrome was considered in $50 \%$ of the cases. All patients had laboratory features found in HELLP syndrome, including transaminitis (not reported by Raucher et al); and thrombocytopenia ranging from $8,000-120,000 / \mu \mathrm{L}$. One patient had proteinuria (case 2). Two patients had coinfection with other tick-borne diseases, namely Lyme disease (case 2) and anaplasmosis. ${ }^{17}$

Our review in pregnancy reinforced that parasitemia load does not necessarily correlate with severity of illness. One patient that had acute hypoxemic respiratory failure had a parasitemia load of just $1.1 \%$ (case 1 ). Laboratory abnormalities and symptomatic infection were present in a patient with a parasitemia load of just $0.08 \%$ (case 2 ). One patient with a parasitemia load of $19.13 \%$ developed severe disseminated intravascular coagulation (DIC), acute kidney infection (AKI), and complications of hemorrhage secondary to DIC (case 3).

Seventy-five percent of the patients were treated with the preferred first line treatment, clindamycin, and quinine. One patient (case 1) had a drug reaction to the regimen; hence, azithromycin and atavoquone were used in her case. Raucher, et al did not utilize antiparasitic agents. One patient with severe babesiosis and parasitemia of $19.13 \%$ required

Table 1 HELLP versus Babesiosis

\begin{tabular}{|l|l|l|}
\hline & HELLP syndrome & Babesiosis \\
\hline $\begin{array}{l}\text { Clinical } \\
\text { Manifestations }\end{array}$ & $\begin{array}{l}\text { Nausea, vomiting, diarrhea, malaise, abdominal pain, } \\
\text { midepigastric pain, headache, jaundice, visual } \\
\text { changes, } \pm \text { elevated blood pressure }\end{array}$ & $\begin{array}{l}\text { Fever, myalgias, arthralgias, anorexia, fatigue, chills/ } \\
\text { sweats, headache, abdominal pain, photophobia }\end{array}$ \\
\hline $\begin{array}{l}\text { Laboratory } \\
\text { findings }\end{array}$ & $\begin{array}{l}\text { Hemolysis, liver enzymes, } \mathrm{LDH}, \mathrm{BUN} / \mathrm{Cr}, \\
\text { Thrombocytopenia, } \pm \text { proteinuria }\end{array}$ & $\begin{array}{l}\text { Hemolysis, liver enzymes, } \mathrm{LDH}, \mathrm{BUN} / \mathrm{Cr}, \\
\text { thrombocytopenia, hematuria, } \pm \text { proteinuria }\end{array}$ \\
\hline Complications & DIC, liver infarction, renal failure, pulmonary edema & $\begin{array}{l}\text { ARDS, DIC, CHF, coma, liver failure, renal failure, } \\
\text { splenic rupture. }\end{array}$ \\
\hline Treatment & Delivery & $\begin{array}{l}\text { Clindamycin/quinine, exchange transfusion for some } \\
\text { patients. }\end{array}$ \\
\hline
\end{tabular}

Abbreviations: ARDS, acute respiratory distress syndrome; BUN, blood urea nitrogen; CHF, congestive heart failure; $\mathrm{Cr}$, creatinine; DIC, disseminated intravascular coagulation; HELLP, hemolysis, elevated liver enzymes, and low platelets; LDH, lactate dehydrogenase. 


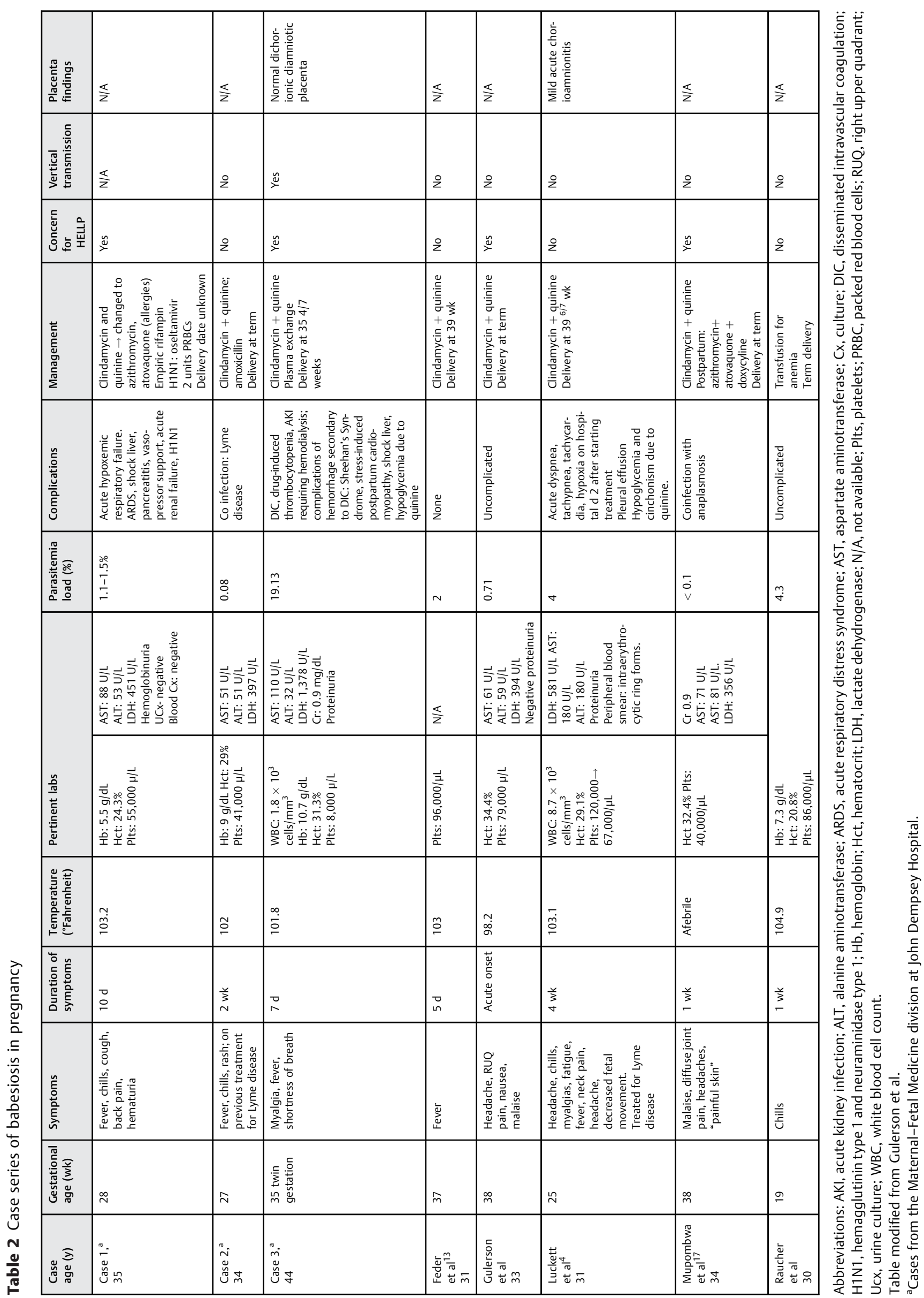


Table 3 Antibiotic regimen and side effects

\begin{tabular}{|c|c|c|}
\hline Regimen & Dosage & Common side effects \\
\hline \multicolumn{3}{|l|}{$\begin{array}{l}{ }^{\mathrm{a}} \text { Clindamycin } \\
\text { and quinine }\end{array}$} \\
\hline Clindamycin & $\begin{array}{l}\text { Oral: } 600 \mathrm{mg} \text { every } 8 \mathrm{~h} \\
\text { Intravenous: } 300-600 \mathrm{mg} \text { every } 6 \mathrm{~h}\end{array}$ & Diarrhea, abdominal pain, hypersensitivity reaction \\
\hline Quinine & $650 \mathrm{mg}$ every $6-8 \mathrm{~h}$ & Hypoglycemia, skin flushing, vision impairment \\
\hline \multicolumn{3}{|c|}{$\begin{array}{l}\text { Atovaquone } \\
\text { and azithromycin }\end{array}$} \\
\hline Atovaquone & $750 \mathrm{mg}$ every $12 \mathrm{~h}$ & Diarrhea, abdominal pain, rash, headache \\
\hline Azithromycin & $\begin{array}{l}500 \mathrm{mg} \text { on } \mathrm{d} 1 \text { followed } \\
\text { by } 25 \mathrm{mg} \text { on subsequent } \mathrm{d}\end{array}$ & Diarrhea, abdominal pain, nausea \\
\hline
\end{tabular}

aPreferred treatment in pregnancy. Modified from CDC, available at: www.cdc.gov/parasites/babesiosis/health_professionals/index.html. Modified from (Vannier and Krause ${ }^{3}$ ).

treatment with plasma exchange in addition to antibiotics (case 3). Two cases required blood transfusion for anemia. Case 1 was lost to follow-up, and all other pregnancies were delivered at term. There was one case of vertical transmission of babesiosis in this series.

\section{Treatment}

\section{Antimicrobial Therapy}

Per CDC guidelines, the standard antibiotic treatment regimen for nonsevere babesiosis in adults is azithromycin plus atovaquone. Clindamycin and quinine is the preferred regimen in pregnant patients and severely ill patients. Drug regimens for both groups are for approximately 7 to 10 days. Clindamycin and quinine provide better placental penetration to prevent vertical transmission; however, atovaquone and azithromycin have also been used to treat malaria in pregnancy without increased risk of congenital birth defects, stillbirth, or anemia. ${ }^{4,17}$ An alternative drug therapy option is critical to note because quinine therapy is often interrupted because of drug toxicity; thus, azithromycin plus atovaquone may be considered appropriate for some hospitalized patients with severe babesiosis. ${ }^{14}$ Antibiotic dosing and side effects are noted in -Table 3.

Persistent or relapsing babesiosis infections may be present in profoundly immunocompromised patients, and treatment can be extended for 6 weeks or longer. Negative blood smears are obtained for 2 weeks or longer before discontinuation. ${ }^{15}$

Current guidelines only advocate treating symptomatic babesiosis patients. ${ }^{15}$ There are no randomized trials or studies to guide the treatment in pregnancy. However, persons with underlying health conditions may have a significant mortality risk with exposure to babesial infection. ${ }^{12}$ As pregnant women are often considered immunocompromised, and evidence also exists in the literature regarding transplacental infection and congenital babesiosis, we would advocate for the treatment of all pregnant women diagnosed with babesiosis, even if asymptomatic. The pediatric team should also be alerted to maternal history of babesiosis so that appropriate blood work and surveillance can be performed after delivery.

\section{Exchange Transfusion}

Exchange transfusion has been recommended by the Infectious Disease Society of America and American Society for Apheresis for patients with high-grade parasitemia $>10 \%$ or in the presence of significant comorbidities including hemolysis, renal, hepatic, or pulmonary compromise. ${ }^{12}$ Exchange transfusion is used in conjunction with antimicrobial agents. ${ }^{18}$ Patients generally tolerate the exchange transfusion well with exposure to multiple red cell transfusions being the most significant risk. ${ }^{12}$ The exchange transfusion is believed to have multiple beneficial effects including removing proinflammatory cytokines and reducing the level of parasitemia. ${ }^{12}$ Because the severity of disease is not always directly related to parasite load, some have suggested consideration for RBC exchange at lower parasite loads, especially in immunocompromised hosts. ${ }^{18}$ There are no data available to determine if partial exchange is preferable to whole blood exchange. Expert consultation with a hematologist and infectious disease experts should be obtained. ${ }^{19}$

\section{Take Home Points}

Babesiosis is a rare tick-borne illness which can be a parasitic imitator of HELLP syndrome. Clinical manifestations and laboratory evaluation may be similar between the two conditions making it difficult to sometimes distinguish between them. Babesiosis should be considered on the differential for patients who live or have traveled to endemic areas of babesiosis or have received a blood transfusion. A blood smear with the presence of intraerythrocytic parasites may help to guide diagnosis in patients with hemolytic anemia. ${ }^{4} \mathrm{~A}$ high index of suspicion is essential to ensure prompt and correct treatment of babesiosis and to avoid iatrogenic preterm delivery potentially. ${ }^{4}$

Conflict of Interest

None.

\section{References}

1 Krause PJ, Edoard G, Vannier P. (2018) Babesiosis: microbiology, epidemiology, and pathogenesis. Available from: https://www. 
uptodate.com/contents/babesiosis-microbiology-epidemiologyand-pathogenesis. Accessed January 8, 2018

2 Babesiosis. (2018) Available from: https://www.cdc.gov/parasites/babesiosis/. Accessed August 1, 2018

3 Vannier E, Krause PJ. Human Babesiosis. N Engl J Med 2012;366 (25):2397-2407

4 Luckett R, Rodriguez W, Katz D. Babesiosis in pregnancy. Obstet Gynecol 2014;124(2 Pt 2, Suppl 1):419-422

5 Gulersen M, Brost BC, Bobrovnikov V, Bornstein E. Acute babesiosis in pregnancy: a novel imitator of hemolysis, elevated liver enzymes, and low platelet count syndrome. Obstet Gynecol 2016; 128(01):197-200

6 Linden JV, Prusinski MA, Crowder LA, et al. Transfusion-transmitted and community-acquired babesiosis in New York, 2004 to 2015. Transfusion 2018;58(03):660-668

7 Kjemtrup AM, Conrad PA. Human babesiosis: an emerging tickborne disease. Int J Parasitol 2000;30(12,13):1323-1337

8 FDA. U.S. (2018) FDA approves first tests to screen for tickborne parasite in whole blood and plasma to protect the U.S. blood supply. Available from: https://www.fda.gov/newsevents/newsroom/press announcements/ucm599782.htm. Accessed August 17, 2018

9 Esernio-Jenssen D, Scimeca PG, Benach JL, Tenenbaum MJ. Transplacental/perinatal babesiosis. J Pediatr 1987;110(04): 570-572

10 Joseph JT, Purtill K, Wong SJ et al. Vertical transmission of Babesia microti, United States. Emerg Infect Dis 2012;18(18):1318-1321
11 Vannier EG, Diuk-Wasser MA, Ben Mamoun C, Krause PJ. Babesiosis. Infect Dis Clin North Am 2015;29(02):357-370

12 Spaete J, Patrozou E, Rich JD, Sweeney JD. Red cell exchange transfusion for babesiosis in Rhode Island. J Clin Apher 2009;24 (03):97-105

13 Feder HM Jr., Lawlor M, Krause PJ. Babesiosis in pregnancy. N Engl J Med 2003;349(02):195-196

14 Hatcher JC, Greenberg PD, Antique J, Jimenez-Lucho VE. Severe babesiosis in Long Island: review of 34 cases and their complications. Clin Infect Dis 2001;32(08):1117-1125

15 Sanchez E, Vannier E, Wormser GP, Hu LT. Diagnosis, treatment, and prevention of lyme disease, human granulocytic anaplasmosis, and babesiosis: a review. JAMA 2016;315(16):1767-1777

16 The American College of Obstetricians and Gynecologists. Hypertension in Pregnancy. Washington, WA: The American College of Obstetricians and Gynecologists; 2013

17 Mupombwa T, Mulla BM, Kirby JO'Brien BM. Babesia microti infection in pregnancy mimicking HELLP syndrome. J Bacteriol Parasitol 2016;7(06):1000297-1000298

18 Evenson DA, Perry E, Kloster B, Hurley R, Stroncek DF. Therapeutic apheresis for babesiosis. J Clin Apher 1998;13(01):32-36

19 Wormser GP, Dattwyler RJ, Shapiro ED, et al. The clinical assessment, treatment, and prevention of lyme disease, human granulocytic anaplasmosis, and babesiosis: clinical practice guidelines by the Infectious Diseases Society of America. Clin Infect Dis 2006; 43(09):1089-1134 